\title{
A New Terminal Converging Adaptive Control for Six Degree of Freedom Parallel Robotic Manipulators with Bounded Control Inputs
}

\author{
Dongya Zhao ${ }^{1 *}$, Sarah K. Spurgeon ${ }^{1,2}$, Hao Liang ${ }^{1}$, Shaoyuan $\mathrm{Li}^{3}$, Quanmin Zhu ${ }^{1,4}$ \\ 1. College of Chemical Engineering, China University of Petroleum, Qingdao 266580, China \\ 2. Department of Electronic and Electrical Engineering, University College London, Torrington Place, London \\ WC1E 7JE, United Kingdom \\ 3. Department of Automation, Shanghai Jiao Tong University, Shanghai 200240, China \\ 4. Department of Engineering Design and Mathematics, University of the West of England, Coldharbour Lane, \\ Bristol BS16 1QY, United Kingdom \\ Corresponding Authors Email: dyzhao@upc.edu.cn; dongyazhao@139.com
}

\begin{abstract}
In this study, a new terminal converging adaptive control approach with bounded control inputs is developed for the 6 degree of freedom (DOF) parallel robot manipulator. The non-smooth feedback control principle is combined with particular bounded functions to define both the control input and associated adaptive law. The Lyapunov method is used to present a stability analysis in order to prove that the error trajectories are semi-globally asymptotically stable. Numerical simulation results relating to a 6 DOF parallel robot are presented to validate the effectiveness of the proposed approach and to compare the performance obtained with other candidate control schemes. It is shown that the proposed scheme achieves more rapid error convergence and exhibits improved robustness whilst guaranteeing that the control signal remains within known bounds.
\end{abstract}

Keywords: Non-smooth control, Saturation control, Robot manipulator, Parallel robot

\section{Introduction}

The 6 DOF parallel robot manipulator has some superior properties when compared with its serial counterpart such as higher accuracy, higher stiffness and higher load-carrying capacity $[1,2]$. By virtue of these merits, they can be used as actuators for high precision operation of heavy payload such as a flight simulator, an astronomical telescope or machine-tools [3, 4]. Such applications require high performance control, which means the designed 
control algorithm should achieve high precision and a fast convergence speed.

From the point of view of systems and control, the 6 DOF parallel robot manipulator is a typical multi-input multi-output (MIMO), strongly coupled nonlinear system. Due to the complex dynamics and application environment, modeling error cannot be avoided. It is very challenging to design high performance control algorithms and this has attracted extensive interest in the control problem from both academia and industry.

Adaptive control algorithms are designed to deal with parameter uncertainties $[5,6]$. Robust control approaches are used to overcome the effects of system uncertainty and of external disturbances [7, 8]. Synchronized control strategies are developed to enhance the control performance of parallel robot manipulators $[9,10]$. All the above mentioned algorithms seek to improve the control performance of parallel robot manipulators but they do not consider the effects of possible saturation of the control input. It should be noted that the actuators of parallel robot manipulators' are typically servo motors or hydraulic cylinders, and these cannot produce unbounded control torque. Therefore, control input saturation is a practical issue of relevance to controller design for parallel robots. The control performance will seriously degrade or the actuators will be damaged if the limitations of the control inputs are not considered appropriately in the algorithm design [11, 12]. In serial robot manipulator control, saturation functions are used in the controller design to avoid control input limitations $[13,14]$. However, the speed of convergence of the tracking error will decrease due to the use of the saturation functions.

Non-smooth control has important advantages such as high precision, rapid speed of convergence and stronger robustness when compared with conventional smooth control methods $[15,16]$. The mentioned merits render the approach particularly appropriate for control of mechanical systems [17-20].

A novel terminal converging control approach has been proposed which uses non-smooth feedback for the $6 \mathrm{DOF}$ parallel robot manipulator [21, 22]. However this approach has not considered control input saturation. By using fractional powers of the tracking error, a novel saturation PD with gravity compensation control approach is proposed for 6 DOF parallel robot manipulators, which improves the control performance [23]. However, this method requires an accurate model of the gravity vector which is difficult to obtain in practice due to parameter uncertainty.

To address the high performance control requirements for 6 DOF parallel robot manipulators with bounded control inputs, this study proposes a new adaptive PD control approach using the Lyapunov method, incorporating a fractional power of the tracking error and the properties of the hyperbolic tangent function. The bounds on the control input can be computed a priori to avoid actuator saturation. The gravity vector can be estimated online by using an adaptive law. By using a fractional power of the tracking error in the controller design, the proposed approach prescribes rapid terminal convergence which prescribes higher precision, faster convergence speed and 
stronger robustness than exhibited by conventional approaches $[13,14]$.

The paper is organized as follows: in Section 2, the problem is formulated and some key properties and definitions are introduced. In Section 3, the proposed control algorithm is described and the stability analysis is presented. Numerical simulation studies are given Section 4 and the proposed approach is compared with the conventional approach. Finally, in Section 5, some concluding remarks are presented.

\section{Problem formulation}

A 6 DOF parallel robot manipulator is composed of two bodies connected by six extendable legs, where the configuration is shown in Figure 1. The work space coordinates of the centre of mass of the moving platform can be written as:

$$
\boldsymbol{q}=\left[\begin{array}{llllll}
X & Y & Z & \beta & \gamma & \delta
\end{array}\right]^{T}
$$

where $X, Y, Z$ denote translations and $\beta, \gamma, \delta$ denote rotations. In terms of the Euler-Lagrange method, the dynamic model of the 6 DOF parallel robot manipulator can be described as $[1,24]$ :

$$
\mathbf{M}(\boldsymbol{q}) \ddot{\boldsymbol{q}}+\mathbf{C}(\boldsymbol{q}, \dot{\boldsymbol{q}}) \dot{\boldsymbol{q}}+\mathbf{G}(\boldsymbol{q})=\mathbf{J}(\boldsymbol{q}) \tau
$$

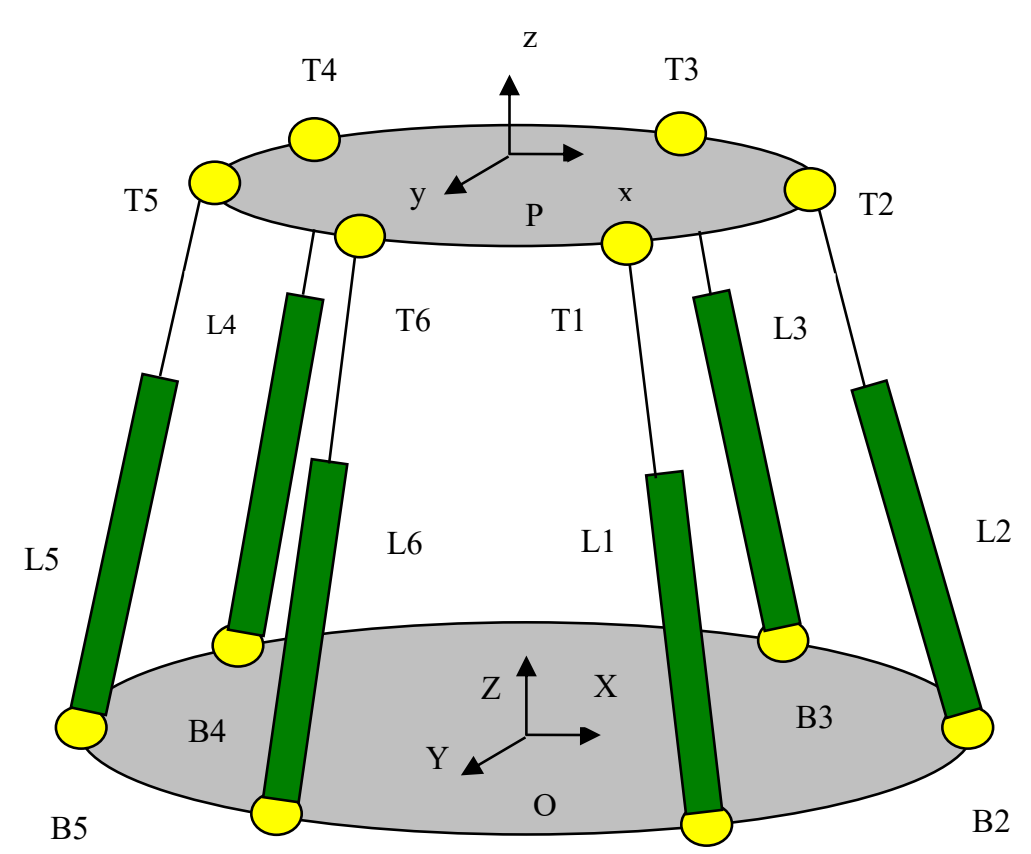

B6

B1

Figure 1 The structure of 6 DOF parallel robot manipulator

where $\mathbf{M}(\boldsymbol{q}) \in R^{6 \times 6}$ is the positive definite symmetric inertia matrix, $\mathbf{C}(\boldsymbol{q}, \dot{\boldsymbol{q}}) \in R^{6 \times 6}$ is the Coriolis and 
centrifugal force vector, $\mathbf{G}(\boldsymbol{q}) \in R^{6}$ is the gravity force vector, $\mathbf{J}(\boldsymbol{q}) \in R^{6 \times 6}$ is the Jacobian matrix and $\tau \in R^{6}$ is the control input.

Notation: For matrix $\mathbf{A} \in R^{n \times n}, \lambda_{\min / \max }$ denotes the minimum/maximum eigenvalue of $\mathbf{A} .\|\cdot\|$ denotes the $L_{2}$ norm for a vector and matrix, $\|\cdot\|_{\infty}$ denotes the infinity norm of a matrix and $\mathbf{I}$ is an appropriately dimensioned identity matrix.

The following properties are assumed:

Property 1: For $\underline{m}<0$ and $\bar{m}>0, \underline{m} \mathbf{I} \leq \mathbf{M}(\boldsymbol{q}) \leq \bar{m} \mathbf{I}$.

Property 2: For $\mu_{M}>0,\|\mathbf{M}(\boldsymbol{q})\| \leq \mu_{M}$.

Property 3: For $\mu_{C}>0,\|\mathbf{C}(\boldsymbol{q}, \dot{\boldsymbol{q}})\| \leq \mu_{C}\|\dot{\boldsymbol{q}}\|$.

Property 4: For $\mu_{G}>0,\|\mathbf{G}(\boldsymbol{q})\| \leq \mu_{G}$.

Property 5: Matrix $\dot{\mathbf{M}}(q)-2 \mathbf{C}(\boldsymbol{q}, \dot{\boldsymbol{q}})$ is skew-symmetric, that is, for $\boldsymbol{x} \in R^{6}, \boldsymbol{x}^{T}[\dot{\mathbf{M}}(\boldsymbol{q})-2 \mathbf{C}(\boldsymbol{q}, \dot{\boldsymbol{q}})] \boldsymbol{x}=0$.

Property 6: The gravity vector can be linearly parameterized as:

$$
\mathbf{G}(\boldsymbol{q})=\mathbf{Y}(\boldsymbol{q}) \boldsymbol{\phi}
$$

where $\mathbf{Y}(\boldsymbol{q}) \in R^{6 \times r}$ is the regression matrix and $\phi \in R^{r}$ is the system parameter vector.

Property 7: For $\zeta_{M}>0,\|\dot{\mathbf{M}}(\boldsymbol{q})\| \leq \zeta_{M}\|\dot{\boldsymbol{q}}\|$.

Property 8: For $\zeta_{J}>0,\left\|\mathbf{J}^{-1}\right\|_{\infty} \leq \zeta_{J}$.

The control objective is to design a terminal converging control algorithm with bounded control input which can achieve semi-global asymptotic stability. The proposed approach can make the tracking error converge to zero as time approaches infinity.

To facilitate the controller design and the corresponding stability analysis, the following definitions are given for $x \in R^{n} \quad[13,14]:$

$$
\begin{gathered}
\operatorname{Tanh}(\boldsymbol{x})=\left[\tanh \left(x_{1}\right), \tanh \left(x_{2}\right), \cdots, \tanh \left(x_{n}\right)\right]^{T} \\
\sqrt{\ln \operatorname{Cosh}(\boldsymbol{x})}=\left[\sqrt{\ln \cosh \left(x_{1}\right)}, \sqrt{\ln \cosh \left(x_{2}\right)}, \cdots, \sqrt{\ln \cosh \left(x_{n}\right)}\right]^{T} \\
\operatorname{Sech}^{2}(\boldsymbol{x})=\left[\operatorname{sech}^{2}\left(x_{1}\right), \operatorname{sech}^{2}\left(x_{2}\right), \cdots, \operatorname{sech}^{2}\left(x_{n}\right)\right]^{T} \\
\operatorname{sig}(\boldsymbol{x})^{\alpha}=\left[\left|x_{1}\right|^{\alpha} \operatorname{sgn}\left(x_{1}\right), \cdots\left|x_{n}\right|^{\alpha} \operatorname{sgn}\left(x_{n}\right)\right]^{T}
\end{gathered}
$$




$$
\begin{gathered}
\int_{0_{n}}^{\Delta x} \operatorname{Tanh}\left(\mathbf{K} * \operatorname{sig}(\boldsymbol{x})^{\alpha}\right) d \boldsymbol{x}=\left[\int_{0}^{\Delta x_{1}} \tanh \left(k_{11}\left|x_{1}\right|^{\alpha} \operatorname{sgn}\left(x_{1}\right)\right) d x_{1}, \cdots, \int_{0}^{\Delta x_{n}} \tanh \left(k_{n n}\left|x_{n}\right|^{\alpha} \operatorname{sgn}\left(x_{n}\right)\right) d x_{n}\right]^{T} \\
\sqrt{\int_{0}^{\Delta x} \operatorname{Tanh}\left(\mathbf{K} \operatorname{sig}(\boldsymbol{x})^{\alpha}\right) d \boldsymbol{x}}=\left[\sqrt{\int_{0}^{\Delta x_{1}} \tanh \left(k_{11}\left|x_{1}\right|^{\alpha} \operatorname{sgn}\left(x_{1}\right)\right) d x_{1}}, \cdots, \sqrt{\int_{0}^{\Delta x_{n}} \tanh \left(k_{n n}\left|x_{n}\right|^{\alpha} \operatorname{sgn}\left(x_{n}\right)\right) d x_{n}}\right]^{T}
\end{gathered}
$$

where $\mathbf{K} \in R^{n \times n}$ is a positive definite diagonal matrix, $k_{i i}, i=1, \cdots, n$ is the $i$ th diagonal element, $x_{i}$ is the $i$ th element of $\boldsymbol{x}$ and $\alpha \in R$.

It is straightforward to show that the following inequalities hold for $x, y \in R^{n}$ and $\psi \in R^{n \times n}$ :

$$
\begin{gathered}
2 \sum_{i=1}^{n} \ln \left(\cosh \left(x_{i}\right)\right) \geq\|\operatorname{Tanh}(\boldsymbol{x})\|^{2} \geq \tanh ^{2}(\|\boldsymbol{x}\|) \\
\boldsymbol{x}^{T} \boldsymbol{T} \operatorname{Tanh}(\boldsymbol{x}) \geq \lambda_{\min }(\boldsymbol{\psi})\|\operatorname{Tanh}(\boldsymbol{x})\|^{2} \\
\|\boldsymbol{x}\|+1 \geq \frac{\|\boldsymbol{x}\|}{\tanh (\|\boldsymbol{x}\|)} \\
\|\boldsymbol{x}\|\|\boldsymbol{y}\| \leq\|\boldsymbol{x}\|^{2}+\|\boldsymbol{y}\|^{2} \\
\|\boldsymbol{x}\| \geq\|\operatorname{Tanh}(\boldsymbol{x})\|
\end{gathered}
$$

\section{Terminal converging adaptive control with bounded inputs}

Several assumptions are first given to facilitate the controller design [1-4]:

Assumption 1: $\boldsymbol{q}$ and $\dot{\boldsymbol{q}}$ are measurable; they can be measured by using an exteroceptive sensor [27].

Assumption 2: The desired set point position is given by $\boldsymbol{q}_{\boldsymbol{d}} \in R^{6}$, for $c_{0}>0,\left\|\boldsymbol{q}_{\boldsymbol{d}}\right\| \leq c_{0}, \dot{\boldsymbol{q}}_{\boldsymbol{d}}=0$.

Assumption 3: The system parameters are bounded as:

$$
\underline{\phi_{i}} \leq \phi_{i} \leq \bar{\phi}_{i}
$$

where $\underline{\phi}_{i}, \bar{\phi}_{i} \in R$ are the $i$ th element of vectors $\underline{\phi}, \bar{\phi} \in R^{r}$, respectively.

Assumption 4: $\|\mathbf{Y}\|_{i \infty} \leq \zeta_{Y}$ for $\zeta_{Y}>0$.

Defining the following tracking error as:

$$
\Delta q=q-q_{d}
$$

According to Properties 1-8 and under Assumptions 1-4, the adaptive control law is designed as follows:

$$
\boldsymbol{\tau}=\mathbf{J}^{-1}\left[\mathbf{Y} \hat{\boldsymbol{\phi}}-\mathbf{L}_{\mathbf{P} 1} \operatorname{Tanh}\left(\mathbf{K}_{\mathbf{P} \mathbf{1}} \Delta \boldsymbol{q}\right)-\mathbf{L}_{\mathbf{P} 2} \operatorname{Tanh}\left(\mathbf{K}_{\mathbf{P} 2} \operatorname{sig}(\Delta \boldsymbol{q})^{\alpha}\right)-\mathbf{L}_{\mathbf{D}} \operatorname{Tanh}\left(\mathbf{K}_{\mathbf{D}} \dot{\boldsymbol{q}}\right)\right]
$$


where $\hat{\phi} \in R^{r}$ is the estimate of $\phi$ and $\mathbf{K}_{\mathbf{P 1}}, \mathbf{K}_{\mathbf{P} 2}, \mathbf{K}_{\mathbf{D}}, \mathbf{L}_{\mathbf{P} 1}, \mathbf{L}_{\mathbf{P} 2}, \mathbf{L}_{\mathbf{D}} \in R^{6 \times 6}$ are positive definite diagonal gain matrices.

Remark 1: If the desired position is selected appropriately, the 6 DOF parallel robot manipulator can operate in a singularity free work space and $\mathbf{J}^{-1}$ will always exist. Recently, a complete set of necessary conditions have been derived for enabling parallel robots to pass through type II singular configurations while maintaining stable motion [28]. This contribution increases the potential applicability of the approach proposed in this paper.

Define the estimation error as:

$$
\tilde{\phi}=\hat{\phi}-\phi
$$

where $\tilde{\phi} \in R^{r}$ denotes the estimation error vector.

The adaptive law is designed as:

$$
\dot{\hat{\boldsymbol{\phi}}}=\dot{\tilde{\boldsymbol{\phi}}}=\operatorname{proj}\{\boldsymbol{\Omega}\}
$$

where $\boldsymbol{\Omega} \in R^{r}$ is the auxiliary term, which is designed as:

$$
\boldsymbol{\Omega}=-\Gamma \mathbf{Y}^{T}(\dot{\boldsymbol{q}}+\varepsilon \operatorname{Tanh}(\Delta \boldsymbol{q}))
$$

where $\Gamma \in R^{r \times r}$ is a positive definite diagonal gain matrix and $\varepsilon \in R$. The function $\operatorname{proj}\{\boldsymbol{\Omega}\}$ is defined as follows [14]:

$$
\operatorname{proj}\left\{\Omega_{i}\right\}=\left\{\begin{array}{lllll}
\Omega_{i} & \text { if } & \hat{\phi}_{i}>\underline{\phi}_{i} & & \\
\Omega_{i} & \text { if } & \hat{\phi}_{i}=\underline{\phi}_{i} & \text { and } & \Omega_{i} \geq 0 \\
0 & \text { if } & \hat{\phi}_{i}=\bar{\phi}_{i} & \text { and } & \Omega_{i}<0 \\
0 & \text { if } & \hat{\phi}_{i}=\bar{\phi}_{i} & \text { and } & \Omega_{i}>0 \\
\Omega_{i} & \text { if } & \hat{\phi}_{i}=\bar{\phi}_{i} & \text { and } & \Omega_{i} \leq 0 \\
\Omega_{i} & \text { if } & \hat{\phi}_{i}<\bar{\phi}_{i} & &
\end{array}\right.
$$

where $\underline{\phi}_{i} \leq \hat{\phi}_{i}(0) \leq \bar{\phi}_{i}, \Omega_{i}$ is the $i$ th elements of $\Omega, i=1, \cdots, 6$.

An adaptive control law has been designed for serial robots with bounded control input [14]. This control law is given by:

$$
\boldsymbol{u}=\mathbf{Y} \hat{\boldsymbol{\phi}}-\mathbf{L}_{\mathbf{P}} \operatorname{Tanh}(\Delta \boldsymbol{q})-\mathbf{L}_{\mathbf{D}} \operatorname{Tanh}(\dot{\boldsymbol{q}})
$$

where $\boldsymbol{u} \in R^{n}$ is the joint torque, $\mathbf{L}_{\mathbf{P}}, \mathbf{L}_{\mathbf{D}} \in R^{n \times n}$ are positive definite diagonal gain matrices, $\mathbf{Y} \in R^{n \times m}$ is the regression matrix and $\hat{\phi} \in R^{m}$ is the estimated system parameter vector which is updated online by using a similar adaptive law (19-21). 
Comparing (17) and (22), the control proposed in (17) is different to that presented in (22) and developed in [13, 14] in the following ways:

- $\mathbf{J}$ appears in (17) however it does not appear in (22). This difference is because the dynamic model of the parallel robot is constructed in the task space, however the dynamic model of the series robot is constructed in joint space.

- $\mathbf{L}_{\mathbf{P} 2} \operatorname{Tanh}\left(\mathbf{K}_{\mathbf{P} 2} \operatorname{sig}(\Delta \boldsymbol{q})^{\alpha}\right)$ is used in (17). For example, if $\Delta q_{i} \in R$, with $\left|\Delta q_{i}\right|<1,\left|\Delta q_{i}\right|^{\alpha}>\left|\Delta q_{i}\right|$, this means that as the tracking error $\Delta q_{i}$ approaches zero, the proposed control involving $\left|\Delta q_{i}\right|^{\alpha}$ produces a larger control signal than the control in (22) involving $\left|\Delta q_{i}\right|$. This means that the proposed control (17) provides terminal convergence characteristics. By incorporating this term, the proposed approach has higher precision, faster convergence speed and stronger robustness when compared to the strategy presented in [14].

- In the control (17) $\mathbf{L}_{\mathbf{P 1}}, \mathbf{L}_{\mathbf{P} 2}, \mathbf{L}_{\mathbf{D}}$ are used to tune the magnitude of the control signal providing an explicit control gain to prescribe any control input limitations. $\mathbf{K}_{\mathbf{P} 1}, \mathbf{K}_{\mathbf{P} 2}, \mathbf{K}_{\mathbf{D}}$ have been embedded in the saturation function $\tanh (\cdot)$ and are used to tune the control signals in the saturation function. However, in (22) only $\mathbf{L}_{\mathbf{P}}, \mathbf{L}_{\mathbf{D}}$ are available to tune the controller. Comparing (22) and (17), the proposed approach includes the additional gains $\mathbf{K}_{\mathrm{P} 1}, \mathbf{K}_{\mathrm{P} 2}, \mathbf{K}_{\mathrm{D}}$ in the controller design which provides greater tuning flexibility.

Substituting (17) into (2), the closed-loop system becomes:

$$
\mathbf{M}(\boldsymbol{q}) \ddot{\boldsymbol{q}}+\mathbf{C}(\boldsymbol{q}, \dot{\boldsymbol{q}}) \dot{\boldsymbol{q}}+\mathbf{G}(\boldsymbol{q})=\mathbf{Y} \hat{\boldsymbol{\phi}}-\mathbf{L}_{\mathbf{P} 1} \operatorname{Tanh}\left(\mathbf{K}_{\mathbf{P} 1} \Delta \boldsymbol{q}\right)-\mathbf{L}_{\mathbf{P} 2} \operatorname{Tanh}\left(\mathbf{K}_{\mathbf{P} 2} \operatorname{sig}(\Delta \boldsymbol{q})^{\alpha}\right)-\mathbf{L}_{\mathbf{D}} \operatorname{Tanh}\left(\mathbf{K}_{\mathrm{D}} \dot{\boldsymbol{q}}\right)
$$

The following result relating to the stability of the proposed scheme can now be presented.

Theorem 1: Under Assumptions 1-4, if the following conditions are satisfied, the closed loop dynamic equation (23) will be semi-globally asymptotically stable, that is, $\lim _{t \rightarrow \infty} \Delta \boldsymbol{q}=0$.

Condition 1: $\bar{m} \varepsilon^{2} \leq \lambda_{\min }\left(\mathbf{L}_{\mathbf{P 1}}\right) \lambda_{\text {min }}\left(\mathbf{K}_{\mathbf{P 1}}^{-1}\right)$

Condition 2: $\varepsilon \leq \frac{\lambda_{\min }\left(\mathbf{L}_{\mathbf{P 1}}\right) \lambda_{\min }\left(\mathbf{K}_{\mathbf{D}}^{-1}\right)}{\lambda_{\min }\left(\mathbf{L}_{\mathbf{D}}\right)}$

Condition 3: $\frac{\lambda_{\min }\left(\mathbf{L}_{\mathbf{D}}\right) \lambda_{\min }\left(\mathbf{K}_{\mathbf{D}}\right)}{2 \varepsilon \xi_{\chi}} \geq\left(1+\sqrt{\frac{V_{M}(0)}{2 \bar{m}}}\right)^{2}$

where $V_{M}$ is specified as: 


$$
\begin{aligned}
V_{M} & =\frac{1}{2} \bar{m}\|\dot{\boldsymbol{q}}\|^{2}+\varepsilon \mu_{M}\left\|\mathbf{K}_{\mathbf{P} 1} \Delta \boldsymbol{q}\right\|\|\dot{\boldsymbol{q}}\|+\lambda_{\max }\left(\mathbf{L}_{\mathbf{P} 1}\right) \lambda_{\max }\left(\mathbf{K}_{\mathbf{P} 1}^{-1}\right)\left\|\sqrt{\ln \operatorname{Cosh}\left(\mathbf{K}_{\mathbf{P} 1} \Delta \boldsymbol{q}\right)}\right\|^{2} \\
& +\lambda_{\max }\left(\mathbf{L}_{\mathbf{P} 2}\right)\left\|\int_{0}^{\Delta q} \operatorname{Tanh}\left(\mathbf{K}_{\mathbf{P} 2} \operatorname{sig}(\boldsymbol{r})^{\alpha}\right) d \boldsymbol{r}\right\|+\frac{1}{2} \lambda_{\max }\left(\Gamma^{-1}\right)\|\tilde{\phi}\|^{2}
\end{aligned}
$$

Proof: To prove stability, the following nonnegative scalar Lyapunov function is selected $[13,14]$ :

$$
\begin{aligned}
V & =\frac{1}{2} \dot{\boldsymbol{q}}^{T} \mathbf{M}(\boldsymbol{q}) \dot{\boldsymbol{q}}+\varepsilon\left(\operatorname{Tanh}\left(\mathbf{K}_{\mathbf{P} 1} \Delta \boldsymbol{q}\right)\right)^{T} \mathbf{M}(\boldsymbol{q}) \dot{\boldsymbol{q}} \\
& +\left(\sqrt{\ln \operatorname{Cosh}\left(\mathbf{K}_{\mathbf{P} 1} \Delta \boldsymbol{q}\right)}\right)^{T} \boldsymbol{L}_{\mathbf{P} 1} \mathbf{K}_{\mathbf{P} 1}^{-1}\left(\sqrt{\ln \operatorname{Cosh}\left(\mathbf{K}_{\mathbf{P} 1} \Delta \boldsymbol{q}\right)}\right) \\
& +\left(\sqrt{\int_{0}^{\Delta q} \operatorname{Tanh}\left(\mathbf{K}_{\mathbf{P} 2} \operatorname{sig}(\boldsymbol{r})^{\alpha}\right) d \boldsymbol{r}}\right)^{T} \boldsymbol{L}_{\mathbf{P} 2}\left(\sqrt{\int_{0}^{\Delta q} \operatorname{Tanh}\left(\mathbf{K}_{\mathbf{P} 2} \operatorname{sig}(\boldsymbol{r})^{\alpha}\right) d \boldsymbol{r}}\right)+\frac{1}{2} \tilde{\boldsymbol{\phi}}^{T} \Gamma^{-1} \tilde{\boldsymbol{\phi}}
\end{aligned}
$$

First, it should be proved that the Lyapunov function (24) is positive definite and bounded. Completing the squares in (24):

$$
\begin{aligned}
V= & \frac{1}{2}\left[\dot{\boldsymbol{q}}+\varepsilon \operatorname{Tanh}\left(\mathbf{K}_{\mathbf{P} 1} \Delta \boldsymbol{q}\right)\right]^{T} \mathbf{M}(\boldsymbol{q})\left[\dot{\boldsymbol{q}}+\varepsilon \operatorname{Tanh}\left(\mathbf{K}_{\mathbf{P} \mathbf{1}} \Delta \boldsymbol{q}\right)\right] \\
& -\frac{1}{2} \varepsilon^{2}\left[\operatorname{Tanh}\left(\mathbf{K}_{\mathbf{P} 1} \Delta \boldsymbol{q}\right)\right]^{T} \mathbf{M}(\boldsymbol{q}) \operatorname{Tanh}\left(\mathbf{K}_{\mathbf{P} 1} \Delta \boldsymbol{q}\right) \\
& +\left(\sqrt{\ln \operatorname{Cosh}\left(\mathbf{K}_{\mathbf{P}} \Delta \boldsymbol{q}\right)}\right)^{T} \mathbf{L}_{\mathbf{P} 1} \mathbf{K}_{\mathbf{P} \mathbf{1}}^{-1}\left(\sqrt{\ln \operatorname{Cosh}\left(\mathbf{K}_{\mathbf{P} 1} \Delta \boldsymbol{q}\right)}\right) \\
& +\left(\sqrt{\int_{0}^{\Delta q} \operatorname{Tanh}\left(\mathbf{K}_{\mathbf{P} 2} \operatorname{sig}(\boldsymbol{r})^{\alpha}\right) d \boldsymbol{r}}\right)^{T} \mathbf{L}_{\mathbf{P} 2}\left(\sqrt{\int_{0}^{\Delta q} \operatorname{Tanh}\left(\mathbf{K}_{\mathbf{P} 2} \operatorname{sig}(\boldsymbol{r})^{\alpha}\right) d \boldsymbol{r}}\right)+\frac{1}{2} \tilde{\boldsymbol{\phi}}^{T} \Gamma^{-1} \tilde{\boldsymbol{\phi}}
\end{aligned}
$$

Considering Property 1 and equation (10), the Lyapunov function (25) satisfies the following inequality:

$$
\begin{aligned}
V \geq & \frac{1}{2} \underline{m}\left\|\dot{\boldsymbol{q}}+\varepsilon \operatorname{Tanh}\left(\mathbf{K}_{\mathbf{P} 1} \Delta \boldsymbol{q}\right)\right\|^{2} \\
& -\frac{1}{2} \varepsilon^{2} \bar{m}\left\|\operatorname{Tanh}\left(\mathbf{K}_{\mathbf{P} 1} \Delta \boldsymbol{q}\right)\right\|^{2}+\frac{1}{2} \lambda_{\min }\left(\mathbf{L}_{\mathbf{P} 1}\right) \lambda_{\min }\left(\mathbf{K}_{\mathbf{P} 1}^{-1}\right)\left\|\operatorname{Tanh}\left(\mathbf{K}_{\mathbf{P} 1} \Delta \boldsymbol{q}\right)\right\|^{2} \\
& +\lambda_{\min }\left(\mathbf{L}_{\mathbf{P} 2}\right)\left\|\int_{\mathbf{0}_{n}}^{\Delta q} \operatorname{Tanh}\left(\mathbf{K}_{\mathbf{P} 2} \operatorname{sig}(\boldsymbol{r})^{\alpha}\right) d \boldsymbol{r}\right\|+\frac{1}{2} \lambda_{\min }\left(\Gamma^{-1}\right)\|\tilde{\boldsymbol{\phi}}\|^{2}=V_{m}
\end{aligned}
$$

If Condition 1 holds, $V \geq 0$. It can be seen that $V=0$ if and only if $\left[\dot{\boldsymbol{q}}^{T}, \Delta \boldsymbol{q}^{T}, \tilde{\boldsymbol{\phi}}^{T}\right]^{T}=\mathbf{0}_{\mathbf{1 2 + m}}$. Hence $V$ is positive definite. Considering Properties 1-2 and equation(14), the Lyapunov function (25) satisfies the following inequality:

$$
\begin{aligned}
V \leq & \frac{1}{2} \bar{m}\|\dot{\boldsymbol{q}}\|^{2}+\varepsilon \mu_{M}\left\|\mathbf{K}_{\mathbf{P} 1} \Delta \boldsymbol{q}\right\|\|\dot{\boldsymbol{q}}\| \\
& +\lambda_{\max }\left(\mathbf{L}_{\mathbf{P} 1}\right) \lambda_{\max }\left(\mathbf{K}_{\mathbf{P} 1}^{-1}\right)\left\|\sqrt{\ln \operatorname{Cosh}\left(\mathbf{K}_{\mathbf{P} 1} \Delta \boldsymbol{q}\right)}\right\|^{2} \\
& +\lambda_{\max }\left(\mathbf{L}_{\mathbf{P} 2}\right)\left\|\int_{0}^{\Delta q} \operatorname{Tanh}\left(\mathbf{K}_{\mathbf{P} 2} \operatorname{sig}(\boldsymbol{r})^{\alpha}\right) d \boldsymbol{r}\right\|+\frac{1}{2} \lambda_{\max }\left(\Gamma^{-1}\right)\|\tilde{\boldsymbol{\phi}}\|^{2}
\end{aligned}
$$

Hence, $V$ is bounded as $V_{m} \leq V \leq V_{M}$.

Differentiating (24) with respect to time yields: 


$$
\begin{aligned}
\dot{V}= & \dot{\boldsymbol{q}}^{T} \mathbf{M}(\boldsymbol{q}) \ddot{\boldsymbol{q}}+\frac{1}{2} \dot{\boldsymbol{q}}^{T} \dot{\mathbf{M}}(\boldsymbol{q}) \dot{\boldsymbol{q}} \\
& +\varepsilon \mathbf{K}_{\mathbf{P} \mathbf{1}} \dot{\boldsymbol{q}}^{T} \operatorname{diag}\left\{\operatorname{Sech}^{2}\left(\mathbf{K}_{\mathbf{P} \mathbf{1}} \Delta \boldsymbol{q}\right)\right\} \mathbf{M}(\boldsymbol{q}) \dot{\boldsymbol{q}}+\varepsilon\left(\operatorname{Tanh}\left(\mathbf{K}_{\mathbf{P} 1} \Delta \boldsymbol{q}\right)\right)^{T} \dot{\mathbf{M}}(\boldsymbol{q}) \dot{\boldsymbol{q}} \\
& +\varepsilon\left(\operatorname{Tanh}\left(\mathbf{K}_{\mathbf{P} \mathbf{1}} \Delta \boldsymbol{q}\right)\right)^{T} \mathbf{M}(\boldsymbol{q}) \ddot{\boldsymbol{q}}+\dot{\boldsymbol{q}}^{T} \mathbf{L}_{\mathbf{P} \mathbf{1}} \operatorname{Tanh}\left(\mathbf{K}_{\mathbf{P} \mathbf{1}} \Delta \boldsymbol{q}\right) \\
& +\dot{\boldsymbol{q}}^{T} \mathbf{L}_{\mathbf{P} \mathbf{2}} \operatorname{Tanh}\left(\mathbf{K}_{\mathbf{P} \mathbf{2}} \operatorname{sig}(\Delta \boldsymbol{q})^{\alpha}\right)+\tilde{\boldsymbol{\phi}}^{T} \Gamma^{-1} \dot{\hat{\boldsymbol{\phi}}}
\end{aligned}
$$

Using Property 5 and considering equation (23), (28) can be written as:

$$
\begin{aligned}
\dot{V}= & -\dot{\boldsymbol{q}}^{T} \mathbf{Y} \boldsymbol{\phi}+\dot{\boldsymbol{q}}^{T} \mathbf{Y} \hat{\boldsymbol{\phi}}-\dot{\boldsymbol{q}}^{T} \mathbf{L}_{\mathbf{P} \mathbf{1}} \operatorname{Tanh}\left(\mathbf{K}_{\mathbf{P} \mathbf{1}} \Delta \boldsymbol{q}\right) \\
& -\dot{\boldsymbol{q}}^{T} \mathbf{L}_{\mathbf{P} \mathbf{2}} \operatorname{Tanh}\left(\mathbf{K}_{\mathbf{P} \mathbf{2}} \operatorname{sig}(\Delta \boldsymbol{q})^{\alpha}\right)-\dot{\boldsymbol{q}}^{T} \mathbf{L}_{\mathbf{D}} \operatorname{Tanh}\left(\mathbf{K}_{\mathbf{D}} \dot{\boldsymbol{q}}\right) \\
& +\varepsilon \mathbf{K}_{\mathbf{P} \mathbf{1}} \dot{\boldsymbol{q}}^{T} \operatorname{diag}\left\{\operatorname{Sech}{ }^{2}\left(\mathbf{K}_{\mathbf{P} \mathbf{1}} \Delta \boldsymbol{q}\right)\right\} \mathbf{M}(\boldsymbol{q}) \dot{\boldsymbol{q}}+\varepsilon\left(\operatorname{Tanh}\left(\mathbf{K}_{\mathbf{P} \mathbf{1}} \Delta \boldsymbol{q}\right)\right)^{T} \dot{\mathbf{M}}(\boldsymbol{q}) \dot{\boldsymbol{q}} \\
& +\varepsilon\left(\operatorname{Tanh}\left(\mathbf{K}_{\mathbf{P} \mathbf{1}} \Delta \boldsymbol{q}\right)\right)^{T}\left[-\mathbf{C} \dot{\boldsymbol{q}}-\mathbf{Y} \boldsymbol{\phi}+\dot{\boldsymbol{q}}^{T} \mathbf{Y} \hat{\boldsymbol{\phi}}\right. \\
& \left.-\dot{\boldsymbol{q}}^{T} \mathbf{L}_{\mathbf{P} \mathbf{1}} \operatorname{Tanh}\left(\mathbf{K}_{\mathbf{P} \mathbf{1}} \Delta \boldsymbol{q}\right)-\dot{\boldsymbol{q}}^{T} \mathbf{L}_{\mathbf{P} \mathbf{2}} \operatorname{Tanh}\left(\mathbf{K}_{\mathbf{P} \mathbf{2}} \operatorname{sig}(\Delta \boldsymbol{q})^{\alpha}\right)-\dot{\boldsymbol{q}}^{T} \mathbf{L}_{\mathbf{D}} \operatorname{Tanh}\left(\mathbf{K}_{\mathbf{D}} \dot{\boldsymbol{q}}\right)\right] \\
& +\dot{\boldsymbol{q}}^{T} \mathbf{L}_{\mathbf{P} \mathbf{1}} \operatorname{Tanh}\left(\mathbf{K}_{\mathbf{P} \mathbf{1}} \Delta \boldsymbol{q}\right)+\dot{\boldsymbol{q}}^{T} \mathbf{L}_{\mathbf{P} \mathbf{2}} \operatorname{Tanh}\left(\mathbf{K}_{\mathbf{P} \mathbf{2}} \operatorname{sig}(\Delta \boldsymbol{q})^{\alpha}\right)+\tilde{\boldsymbol{\phi}}^{T} \boldsymbol{\Gamma}^{-1} \dot{\hat{\boldsymbol{\phi}}}
\end{aligned}
$$

Let $\chi=\mathbf{K}_{\mathbf{P} \mathbf{1}} \dot{\boldsymbol{q}}^{T} \operatorname{diag}\left\{\operatorname{Sech}^{2}\left(\mathbf{K}_{\mathbf{P} \mathbf{1}} \Delta \boldsymbol{q}\right)\right\} \mathbf{M}(\boldsymbol{q}) \dot{\boldsymbol{q}}+\varepsilon\left(\operatorname{Tanh}\left(\mathbf{K}_{\mathbf{P} \mathbf{1}} \Delta \boldsymbol{q}\right)\right)^{T}(\dot{\mathbf{M}}(\boldsymbol{q}) \dot{\boldsymbol{q}}-\mathbf{C} \dot{\boldsymbol{q}})$, (29) can be written as:

$$
\begin{aligned}
\dot{V}= & -\dot{\boldsymbol{q}}^{T} \mathbf{L}_{\mathbf{D}} \operatorname{Tanh}\left(\mathbf{K}_{\mathbf{D}} \dot{\boldsymbol{q}}\right)+\varepsilon \chi \\
& -\varepsilon\left(\operatorname{Tanh}\left(\mathbf{K}_{\mathbf{P} \mathbf{1}} \Delta \boldsymbol{q}\right)\right)^{T} \mathbf{L}_{\mathbf{P} \mathbf{1}} \operatorname{Tanh}\left(\mathbf{K}_{\mathbf{P} \mathbf{1}} \Delta \boldsymbol{q}\right) \\
& -\varepsilon\left(\operatorname{Tanh}\left(\mathbf{K}_{\mathbf{P} \mathbf{1}} \Delta \boldsymbol{q}\right)\right)^{T} \mathbf{L}_{\mathbf{P} \mathbf{2}} \operatorname{Tanh}\left(\mathbf{K}_{\mathbf{P} \mathbf{2}} \operatorname{sig}(\Delta \boldsymbol{q})^{\alpha}\right) \\
& -\varepsilon\left(\operatorname{Tanh}\left(\mathbf{K}_{\mathbf{P} \mathbf{1}} \Delta \boldsymbol{q}\right)\right)^{T} \mathbf{L}_{\mathbf{D}} \operatorname{Tanh}\left(\mathbf{K}_{\mathbf{D}} \dot{\boldsymbol{q}}\right)+\tilde{\boldsymbol{\phi}}^{T} \Gamma^{-1}(\dot{\hat{\boldsymbol{\phi}}}-\boldsymbol{\Omega})
\end{aligned}
$$

The above equality satisfies:

$$
\begin{aligned}
\dot{V} \leq & -\lambda_{\text {min }}\left(\mathbf{L}_{\mathbf{D}}\right) \dot{\boldsymbol{q}}^{T} \operatorname{Tanh}\left(\mathbf{K}_{\mathbf{D}} \dot{\boldsymbol{q}}\right)+\varepsilon \chi \\
& -\varepsilon \lambda_{\text {min }}\left(\mathbf{L}_{\mathbf{P} 1}\right) \lambda_{\text {min }}\left(\operatorname{Tanh}\left(\mathbf{K}_{\mathbf{P} \mathbf{1}} \Delta \boldsymbol{q}\right)\right)^{T} \operatorname{Tanh}\left(\mathbf{K}_{\mathbf{P} \mathbf{1}} \Delta \boldsymbol{q}\right) \\
& -\varepsilon \lambda_{\text {min }}\left(\mathbf{L}_{\mathbf{D}}\right)\left(\operatorname{Tanh}\left(\mathbf{K}_{\mathbf{P} \mathbf{1}} \Delta \boldsymbol{q}\right)\right)^{T} \operatorname{Tanh}\left(\mathbf{K}_{\mathbf{D}} \dot{\boldsymbol{q}}\right)
\end{aligned}
$$

Let $a=\lambda_{\text {min }}\left(\mathbf{L}_{\mathbf{D}}\right), b=\varepsilon \lambda_{\text {min }}\left(\mathbf{L}_{\mathbf{P 1}}\right)$ and $c=\varepsilon \lambda_{\text {min }}\left(\mathbf{L}_{\mathbf{D}}\right),(31)$ can be written as:

$$
\begin{aligned}
\dot{V} \leq & -a \dot{\boldsymbol{q}}^{T} \operatorname{Tanh}\left(\mathbf{K}_{\mathbf{D}} \dot{\boldsymbol{q}}\right)+\varepsilon \chi-b\left(\operatorname{Tanh}\left(\mathbf{K}_{\mathbf{P} \mathbf{1}} \Delta \boldsymbol{q}\right)\right)^{T} \operatorname{Tanh}\left(\mathbf{K}_{\mathbf{P} \mathbf{1}} \Delta \boldsymbol{q}\right) \\
& -c\left(\operatorname{Tanh}\left(\mathbf{K}_{\mathbf{P} \mathbf{1}} \Delta \boldsymbol{q}\right)\right)^{T} \operatorname{Tanh}\left(\mathbf{K}_{\mathbf{D}} \dot{\boldsymbol{q}}\right)
\end{aligned}
$$

Considering Properties 3 and 7 and inequalities (4-8), it is obvious that $\|\chi\| \leq \xi_{\chi}\|\dot{\boldsymbol{q}}\|^{2}$, then (32) can be written as: 


$$
\begin{gathered}
\dot{V} \leq-a \lambda_{\min }\left(\mathbf{K}_{\mathbf{D}}^{-1}\right)\left\|\operatorname{Tanh}\left(\mathbf{K}_{\mathbf{D}} \dot{\boldsymbol{q}}\right)\right\|^{2}+\varepsilon \xi_{\chi}\|\dot{\boldsymbol{q}}\|^{2}-\frac{b}{2}\left\|\operatorname{Tanh}\left(\mathbf{K}_{\mathbf{P} \mathbf{1}} \Delta \boldsymbol{q}\right)\right\|^{2} \\
-\frac{b}{2}\left\|\operatorname{Tanh}\left(\mathbf{K}_{\mathbf{P} \mathbf{1}} \Delta \boldsymbol{q}\right)+\frac{c}{b} \operatorname{Tanh}\left(\mathbf{K}_{\mathbf{D}} \dot{\boldsymbol{q}}\right)\right\|^{2}+\frac{c^{2}}{2 b}\left\|\operatorname{Tanh}\left(\mathbf{K}_{\mathbf{D}} \dot{\boldsymbol{q}}\right)\right\|^{2} \\
\dot{V} \leq-\frac{a}{2} \lambda_{\min }\left(\mathbf{K}_{\mathbf{D}}^{-1}\right)\left\|\operatorname{Tanh}\left(\mathbf{K}_{\mathbf{D}} \dot{\boldsymbol{q}}\right)\right\|^{2}+\varepsilon \xi_{\chi}\|\dot{\boldsymbol{q}}\|^{2}-\frac{b}{2}\left\|\operatorname{Tanh}\left(\mathbf{K}_{\mathbf{P} \mathbf{1}} \Delta \boldsymbol{q}\right)\right\|^{2} \\
-\frac{b}{2}\left\|\operatorname{Tanh}\left(\mathbf{K}_{\mathbf{P} \mathbf{1}} \Delta \boldsymbol{q}\right)+\frac{c}{b} \operatorname{Tanh}\left(\mathbf{K}_{\mathbf{D}} \dot{\boldsymbol{q}}\right)\right\|^{2}-\left(\frac{a}{2} \lambda_{\min }\left(\mathbf{K}_{\mathbf{D}}^{-1}\right)-\frac{c^{2}}{2 b}\right)\left\|\operatorname{Tanh}\left(\mathbf{K}_{\mathbf{D}} \dot{\boldsymbol{q}}\right)\right\|^{2}
\end{gathered}
$$

If the following inequalities hold, $\dot{V} \leq 0$ :

$$
\begin{gathered}
\frac{a}{2} \lambda_{\min }\left(\mathbf{K}_{\mathrm{D}}^{-1}\right)-\frac{c^{2}}{2 b} \leq 0 \\
-\frac{a}{2}\left\|\operatorname{Tanh}\left(\mathbf{K}_{\mathrm{D}} \dot{\boldsymbol{q}}\right)\right\|^{2}+\varepsilon \xi_{\chi}\|\dot{\boldsymbol{q}}\|^{2} \leq 0
\end{gathered}
$$

It is obvious that if Condition 2 holds, (35) will be satisfied. In terms of (12), if the following inequality holds, (36) will also hold:

$$
\frac{a}{2 \varepsilon \xi_{\chi}} \geq(1+\|\dot{\boldsymbol{q}}\|)^{2}
$$

If Condition 3 is satisfied, (37) will hold and $\dot{V} \leq 0$. Thus the closed loop system (23) is semi-globally stable under Conditions 1-3. Let $\boldsymbol{y}=\left[\left(\operatorname{Tanh}\left(\mathbf{K}_{\mathbf{D}} \dot{\boldsymbol{q}}\right)\right)^{T},\left(\operatorname{Tanh}\left(\mathbf{K}_{\mathbf{P} 1} \Delta \boldsymbol{q}\right)\right)^{T}\right]^{T}$, there exists $\beta>0$ such that the following inequality holds:

$$
\dot{V} \leq-\beta\|\boldsymbol{y}\|^{2}
$$

Because $\dot{V} \leq 0, V \in L_{\infty}$. Then, $\dot{\boldsymbol{q}}, \Delta \boldsymbol{q}, \boldsymbol{\phi}, \boldsymbol{y} \in L_{\infty}$. Due to $\boldsymbol{\Delta} \boldsymbol{q} \in L_{\infty}$ and $\boldsymbol{q}_{\boldsymbol{d}}$ is bounded, $\boldsymbol{q} \in L_{\infty}$. According to (15) and (18-21), $\dot{\hat{\phi}}, \hat{\boldsymbol{\phi}}, \tau \in L_{\infty}$. In light of Property 1 and (23), $\ddot{\boldsymbol{q}}, \dot{\boldsymbol{y}} \in L_{\infty}$. Hence $\boldsymbol{y}$ is uniformly continuous. By using Barbalat's Lemma [25], $\lim _{t \rightarrow \infty}\|\boldsymbol{y}(t)\|=0$, then $\lim _{t \rightarrow \infty} \Delta \boldsymbol{q}=0$.

The bound on the control input $\tau$ is given by:

$$
\|\boldsymbol{\tau}\| \leq\left\|\mathbf{J}^{-1}\right\|_{i \infty}\left(\|\mathbf{Y}\|_{i \infty \infty}\|\bar{\phi}\|+\lambda_{\max }\left(\mathbf{L}_{\mathbf{P 1}}\right)+\lambda_{\max }\left(\mathbf{L}_{\mathbf{P} \mathbf{2}}\right)+\lambda_{\max }\left(\mathbf{L}_{\mathbf{P D}}\right)\right)
$$

In the controller design the bound on $\tau$ can be determined from $\lambda_{\max }\left(\mathbf{L}_{\mathbf{P 1}}\right), \lambda_{\max }\left(\mathbf{L}_{\mathbf{P} 2}\right)$ and $\lambda_{\max }\left(\mathbf{L}_{\mathbf{P D}}\right)$.

Remark 2: The Lyapunov function and the corresponding stability analysis are different from the analysis in [14]. The fractional power of the tracking error is used in particular to enhance the control performance in this paper.

Remark 3: The contribution of $\lambda_{\text {min }}\left(\mathbf{L}_{\mathbf{P} 2}\right)\left\|\int_{0}^{\Delta P} \operatorname{Tanh}\left(\mathbf{K}_{\mathbf{P} 2} \operatorname{sig}(\boldsymbol{r})^{\alpha}\right) d \boldsymbol{r}\right\|$ to the positive definite of $V$ and the 
contribution of $-\varepsilon\left(\operatorname{Tanh}\left(\mathbf{K}_{\mathbf{P} 1} \Delta \boldsymbol{q}\right)\right)^{T} \mathbf{L}_{\mathbf{P} 2} \operatorname{Tanh}\left(\mathbf{K}_{\mathbf{P} 2} \operatorname{sig}(\Delta \boldsymbol{q})^{\alpha}\right) \leq 0$ to $V \leq 0$ is not explicitly considered. Hence, conservation exists in the controller design. This does not however affect the stability of the closed loop. According to Conditions 1-3 and by using a trial and error method, one can find a set of appropriate controller parameters.

Remark 4: If $\mathbf{J}^{-1}=\mathbf{I}$, the proposed approach can be used to control a serial robot manipulator. Further, if $\alpha=1$, $\mathbf{K}_{\mathbf{P} 1}=\mathbf{K}_{\mathbf{P} 2}=\mathbf{K}_{\mathbf{D}}=\mathbf{I}, \mathbf{L}_{\mathbf{P} 1}+\mathbf{L}_{\mathbf{P} 2}=\mathbf{K}_{\mathbf{P}}, \mathbf{L}_{\mathbf{D}}=\mathbf{K}_{\mathbf{D}}$, (17) reduces to (22), which shows that (22) is a special case of (17). The proposed approach is more general than the one in [14].

\section{Numerical illustration}

In this section, a 6 DOF Stewart Platform is used to test the proposed approach [26]. The system is modeled using SimMechanics in Matlab. The parameters are given as follows: the mass and mass moment of inertia values of upper platform are $m=1216.9 \mathrm{~kg}, I_{X}, I_{Y}\left(I_{Z}\right)=304.48(608.46) \mathrm{kg} \cdot \mathrm{m}^{2}$, the mass moment of inertia values of the upper and lower part of the $i$ th leg are $I_{u X i}, I_{u Y i}\left(I_{u Z i}\right)=24.17(0.023) \mathrm{kg} \cdot \mathrm{m}^{2}$ and $I_{d X}, I_{d Y}\left(I_{d Z}\right)=43.02(0.156) \mathrm{kg} \cdot \mathrm{m}^{2}$, the mass of the upper/ lower part of the $i$ th leg are $\left(m_{u}\right)_{i} /\left(m_{d}\right)_{i}=51.81 / 92.11 \mathrm{~kg}$. In the simulation, it is assumed that the actuator control input for each leg is bounded by $\left|\tau_{i}\right| \leq 5 \times 10^{5} \mathrm{~N}$. The desired position is chosen to be $\boldsymbol{q}_{\boldsymbol{d}}=\left[\begin{array}{llllll}0.3 \mathrm{~m} & 0.2 \mathrm{~m} & 0.6 \mathrm{~m} & 0.2 \mathrm{rad} & 0.5 \mathrm{rad} & 1 \mathrm{rad}\end{array}\right]^{T}$ and the initial position is given by $\boldsymbol{q}=\left[\begin{array}{llllll}0.6 \mathrm{~m} & 0.4 \mathrm{~m} & 1.2 \mathrm{~m} & 0.4 \mathrm{rad} & 1 \mathrm{rad} & 2 \mathrm{rad}\end{array}\right]$. The Jacobian matrix is given in the Appendix and further details can be found in $[29,30]$. The estimated parameters are selected as the mass of upper platform and the mass of the upper/ lower part of leg 1 , whose bounds are given as: $\underline{\phi}_{1}=1000, \underline{\phi}_{2}=62, \underline{\phi_{3}}=31, \bar{\phi}_{1}=1300, \bar{\phi}_{2}=108$, $\bar{\phi}_{3}=72$. The initial value of the estimated parameters are selected as: $\phi_{1}(0)=1000, \phi_{2}(0)=62, \phi_{3}(0)=31$. The performance of the proposed terminal converging adaptive control (TCAC) was compared with the existing adaptive control (AC) [14] to validate its effectiveness. The control parameters are given in Table 1. From Table 1 it is clear that the parameter selection supports a fair comparison.

Table 1 Controller parameters

\begin{tabular}{|l|l|}
\hline TCAC & $\mathbf{L}_{\mathbf{P} 1}=\operatorname{diag}\left\{1 \times 10^{6}\right\}, \quad \mathbf{L}_{\mathbf{P} 2}=\operatorname{diag}\left\{1 \times 10^{6}\right\}, \quad \mathbf{L}_{\mathbf{D}}=\operatorname{diag}\left\{4.5 \times 10^{4}\right\}, \quad \mathbf{K}_{\mathbf{P} 1}=\operatorname{diag}\{1\}$, \\
& $\mathbf{K}_{\mathbf{P} 2}=\operatorname{diag}\{1\}, \mathbf{K}_{\mathbf{D}}=\operatorname{diag}\{1\}, \alpha=0.6, \Gamma=\operatorname{diag}\{5\}, \varepsilon=500$ \\
\hline $\mathrm{AC}$ & $\mathbf{L}_{\mathbf{P}}=\operatorname{diag}\left\{2 \times 10^{6}\right\}, \mathbf{L}_{\mathbf{D}}=\operatorname{diag}\left\{4.5 \times 10^{4}\right\}, \Gamma=\operatorname{diag}\{5\}, \varepsilon=500$ \\
\hline
\end{tabular}


Figure 2 shows the tracking errors in the 6 DOF robot system, where the solid line denotes the AC performance and the dashed line describes the TCAC performance. It is clear that the TCAC has a more rapid speed of convergence than the AC implementation. Figure 3 shows the control input. Neither the TCAC nor the AC exceed the control input limitation. Figures 2 and 3 validate that the TCAC has better performance, particularly in the terminal converging phase. This can be attributed to the nonsmooth feedback term $\mathbf{K}_{\mathbf{P}_{2}} s i g(\Delta q)^{\alpha}$ which prescribes a rapid terminal convergence capacity. Figure 4 illustrates the parameter estimates obtained from the adaptive law for both the TCAC and AC. The estimates are all bounded. The simulation results confirm the effectiveness of the proposed approach.

To further test the proposed approach, an impulsive external disturbance was added to the actuators during the time period $0.4-0.41 \mathrm{~s}$ with amplitude $50000 \mathrm{~N}$. Figure 5 shows the position tracking error and Figure 6 the control input in this case. Both of the approaches exhibit a bounded control input which does not exceed the actuator limitations. Again, the simulation results show that the TCAC exhibits better control performance than the AC.

To summarise, the simulation studies demonstrate that the proposed TCAC provides more rapid convergence and higher control precision in addition to improved robustness properties. 

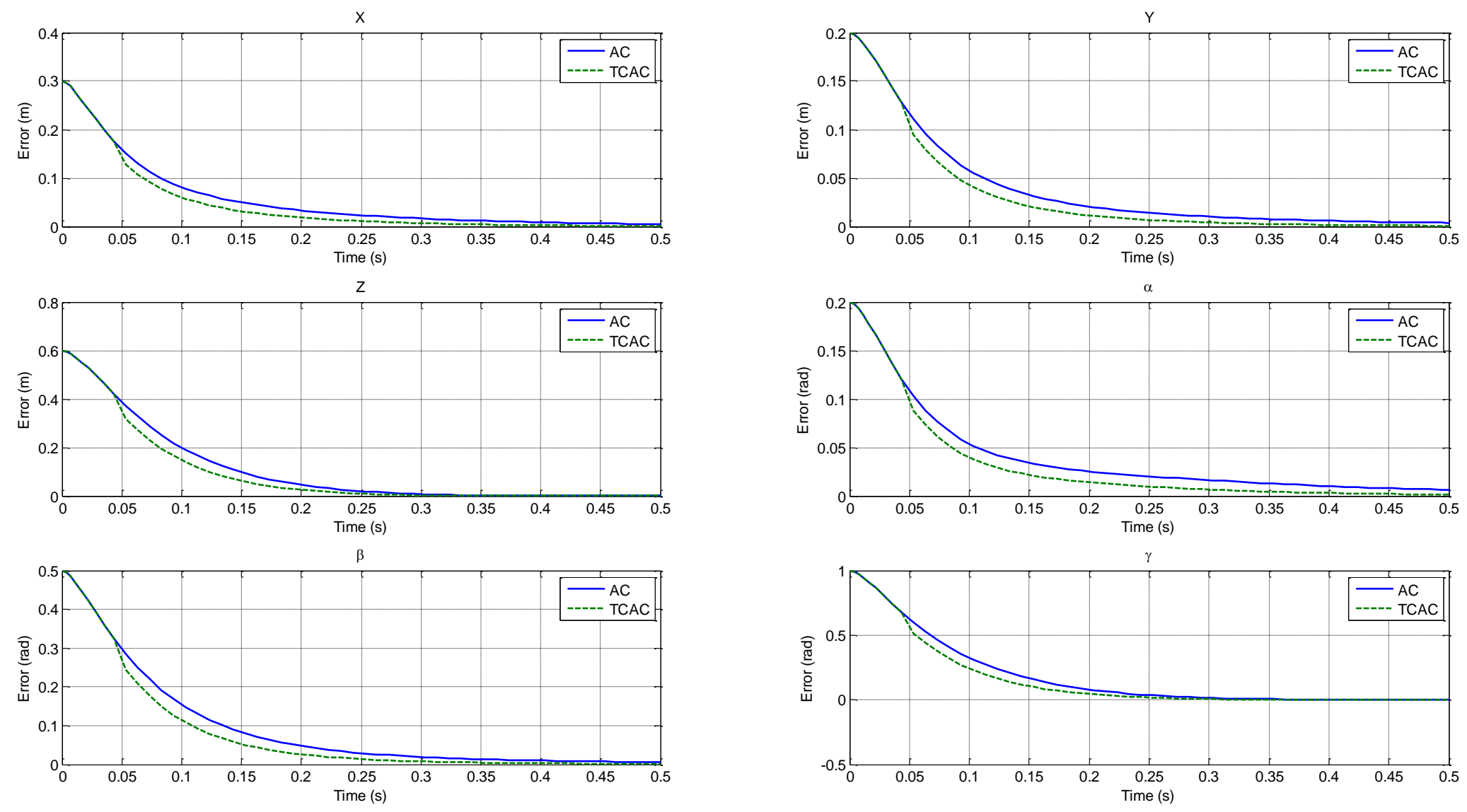

Figure 2 Position tracking errors in the absence of an external disturbance 

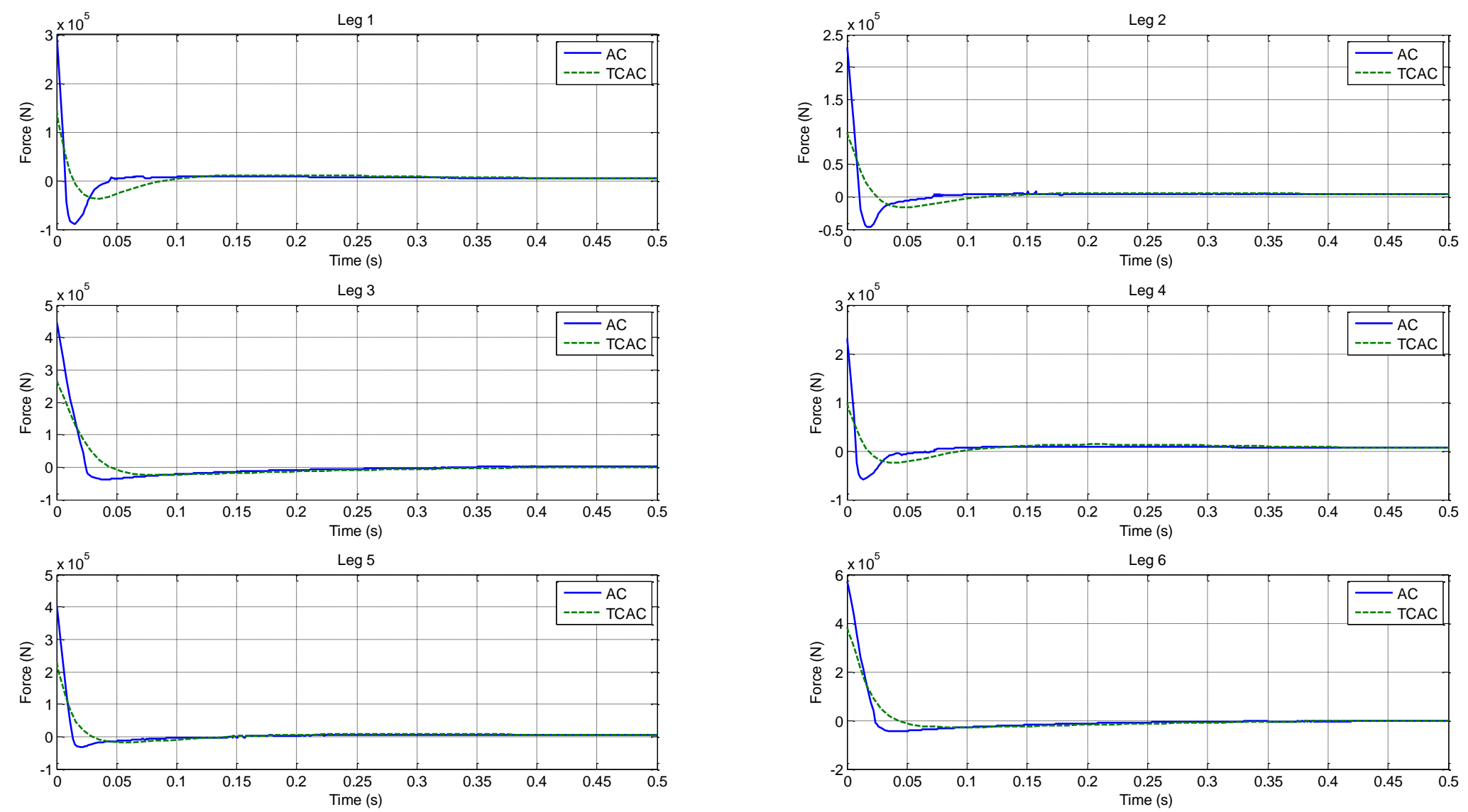

Figure 3 Control input in the absence of an external disturbance 

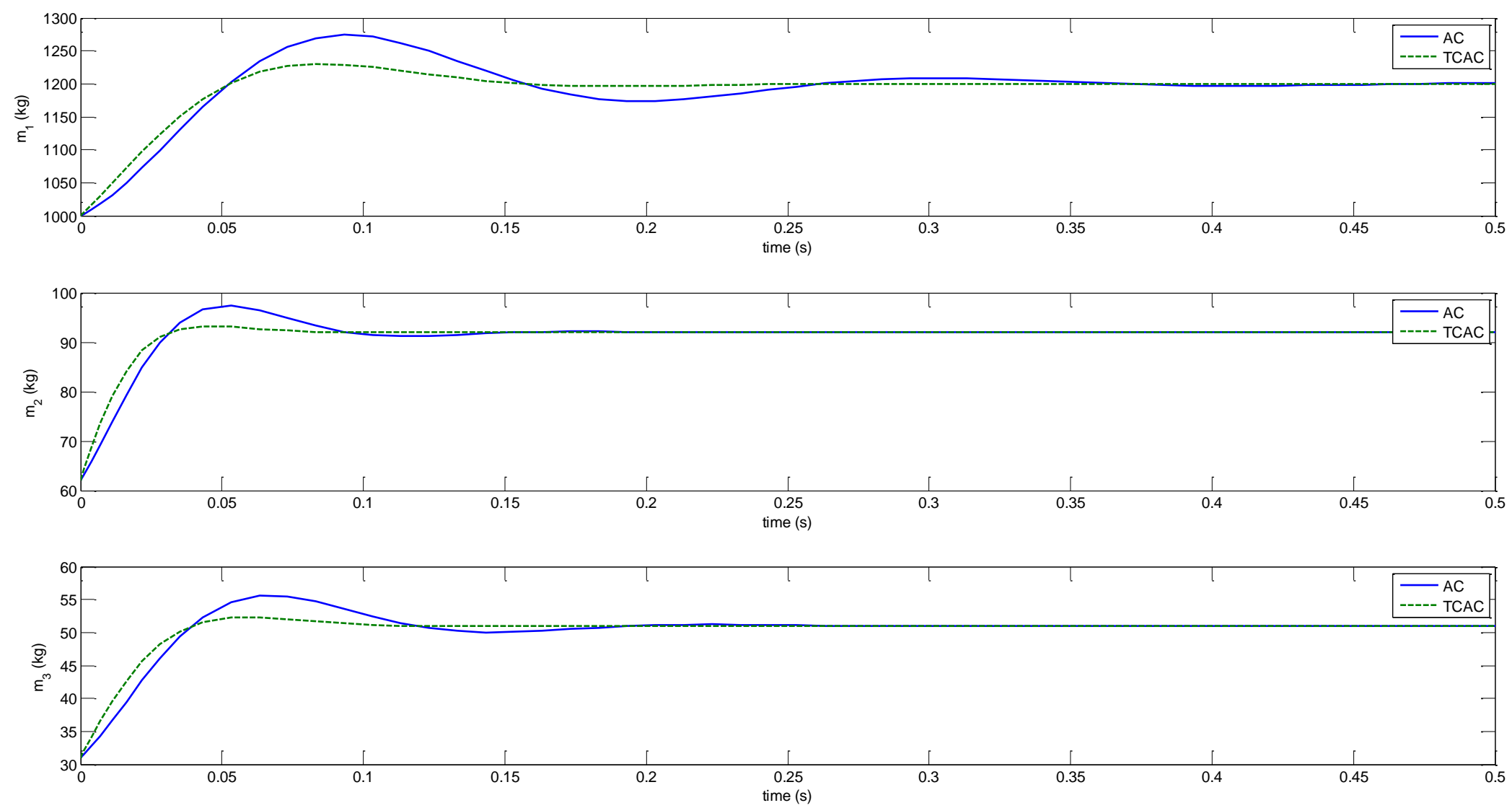

Figure 4 Performance of the adaptive law 



Figure 5 Position tracking errors in the presence of an impulsive external disturbance applied at time 0.4 seconds. 

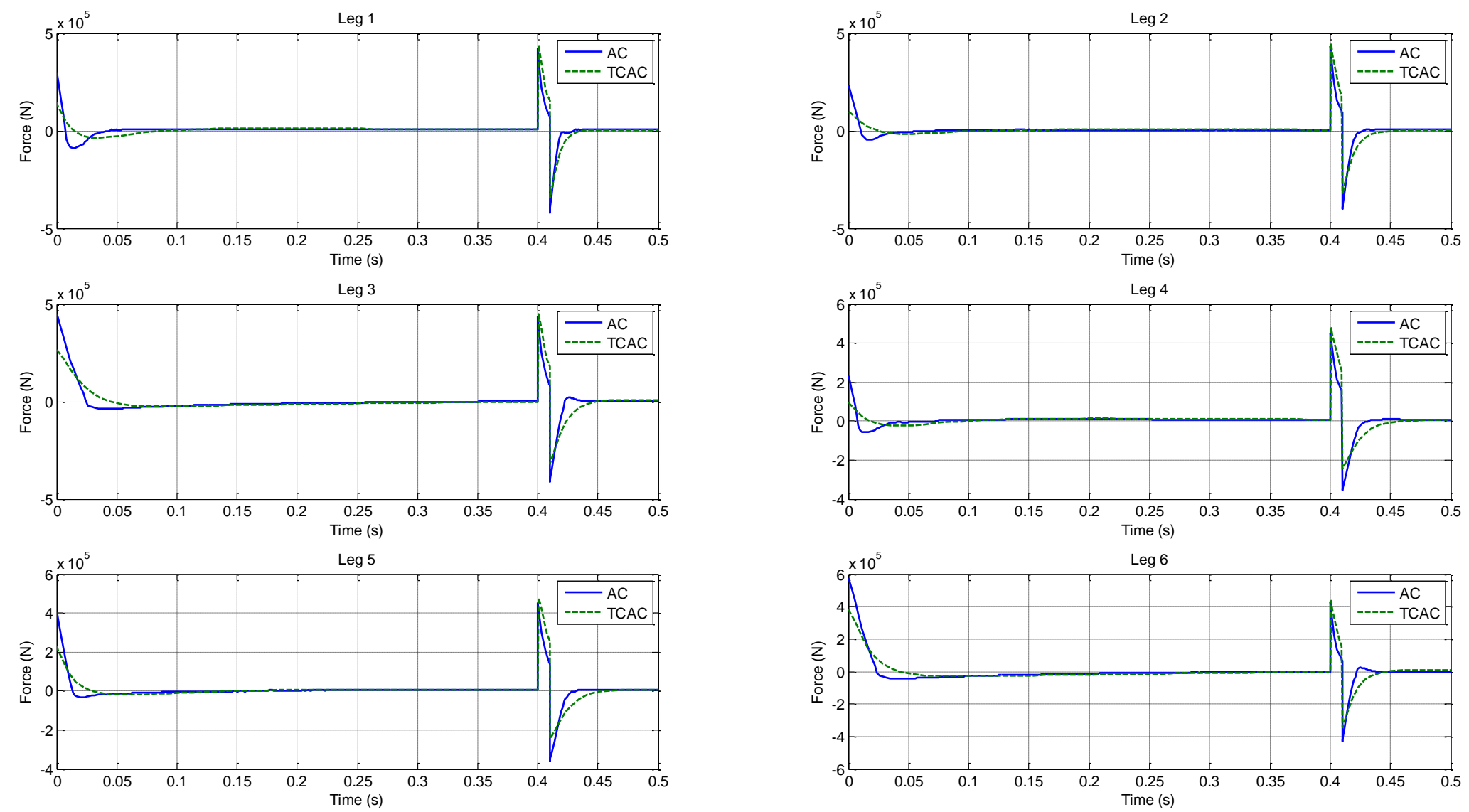

Figure 6 Control input in the presence of an impulsive external disturbance applied at time 0.4 seconds. 


\section{Conclusion}

By resorting to the hyperbolic tangent function and nonsmooth feedback, a novel terminal converging adaptive control with bounded control input has been developed. The proposed approach can achieve semi-global asymptotic stability and provides an effective control solution for parallel robot manipulators. Theoretical analysis and numerical simulation results have been presented to support the arguments presented in the paper. Comprehensive bench tests are now required to further develop and assess the proposed approach. Future work will consider implementation on an industrial manipulator and will assess the trade-off between complexity of implementation and performance by comparing the proposed scheme with the control scheme implemented commercially for common tasks.

\section{Acknowledgements}

This work is partially supported by the National Nature Science Foundation of China under Grant 61473312, 61273188 and the Chang Jiang Scholar Programme. Finally, the authors are grateful to the editor and the anonymous reviewers for their helpful comments and constructive suggestions with regard to the revision of the paper.

\section{Appendix}

The Jacobian matrix for a general six degree of freedom parallel robotic is given by the following equations where full details can be found in $[29,30]$

$$
\begin{gathered}
L_{1}=\sqrt{\left(X_{T 1}-d / 2 \sqrt{3}-b / \sqrt{3}\right)^{2}+\left(Y_{T 1}-d / 2\right)^{2}+Z_{T 1}^{2}} \\
L_{2}=\sqrt{\left(X_{T 1}-d / 2 \sqrt{3}+b / 2 \sqrt{3}\right)^{2}+\left(Y_{T 1}-d / 2-b / 2\right)^{2}+Z_{T 1}^{2}} \\
L_{3}=\sqrt{\left(X_{T 2}+d / \sqrt{3}+b / 2 \sqrt{3}\right)^{2}+\left(Y_{T 2}-b / 2\right)^{2}+Z_{T 2}^{2}} \\
L_{4}=\sqrt{\left(X_{T 2}+d / \sqrt{3}+b / 2 \sqrt{3}\right)^{2}+\left(Y_{T 2}+b / 2\right)^{2}+Z_{T 2}^{2}} \\
L_{5}=\sqrt{\left(X_{T 3}-d / 2 \sqrt{3}+b / 2 \sqrt{3}\right)^{2}+\left(Y_{T 3}+b / 2+d / 2\right)^{2}+Z_{T 3}^{2}} \\
L_{6}=\sqrt{\left(X_{T 3}-d / 2 \sqrt{3}-b / \sqrt{3}\right)^{2}+\left(Y_{T 3}+d / 2\right)^{2}+Z_{T 3}^{2}}
\end{gathered}
$$




$$
\begin{gathered}
X_{T 1}=p_{X}+\frac{a}{\sqrt{3}}\left[\sin \beta \sin \gamma \sin \left(\delta+\frac{\pi}{3}\right)+\cos \gamma \cos \left(\delta+\frac{\pi}{3}\right)\right] \\
Y_{T 1}=p_{Y}+\frac{a}{\sqrt{3}} \cos \beta \sin \left(\delta+\frac{\pi}{3}\right) \\
Z_{T 1}=p_{Z}+\frac{a}{\sqrt{3}}\left[\sin \beta \sin \gamma \sin \left(\delta+\frac{\pi}{3}\right)-\sin \gamma \cos \left(\delta+\frac{\pi}{3}\right)\right] \\
X_{T 2}=p_{X}-\frac{a}{\sqrt{3}}[\sin \beta \sin \gamma \sin \delta+\cos \gamma \cos \delta] \\
Y_{T 2}=p_{Y}-\frac{a}{\sqrt{3}} \cos \beta \sin \delta \\
Z_{T 3}=p_{Z}+\frac{a}{\sqrt{3}}\left[\sin \beta \cos \gamma \sin \left(\delta-\frac{\pi}{3}\right)-\sin \gamma \cos \left(\delta-\frac{\pi}{3}\right)\right] \\
X_{T 3}=p_{X}+\frac{a}{\sqrt{3}}\left[\sin \beta \sin \gamma \sin \left(\delta-\frac{\pi}{3}\right)+\cos \gamma \cos \left(\delta-\frac{\pi}{3}\right)\right] \\
Y_{T 3}=p_{Y}+\frac{a}{\sqrt{3}} \cos \beta \sin \left(\delta-\frac{\pi}{3}\right) \\
\sin \gamma \cos \delta]
\end{gathered}
$$

If $i=1,2$ :

$$
\begin{gathered}
j_{i, 4}=\left(Y_{T 1}-p_{Y}\right)\left(\frac{\partial L_{i}}{\partial X_{T 1}} \sin \gamma+\frac{\partial L_{i}}{\partial Z_{T 1}} \cos \gamma\right)-\frac{\partial L_{i}}{\partial Y_{T 1}} \frac{a}{\sqrt{3}} \sin \beta \sin \left(\delta+\frac{\pi}{3}\right) \\
j_{i, 5}=\left(Z_{T 1}-p_{Z}\right) \frac{\partial L_{i}}{\partial X_{T 1}}-\left(X_{T 1}-p_{X}\right) \frac{\partial L_{i}}{\partial Z_{T 1}} \\
j_{i, 6}=\frac{1}{\sqrt{3}}\left[\frac{\partial L_{i}}{\partial X_{T 1}}\left(X_{T 2}-X_{T 3}\right)+\frac{\partial L_{i}}{\partial Y_{T 1}}\left(Y_{T 2}-Y_{T 3}\right)+\frac{\partial L_{i}}{\partial Z_{T 1}}\left(Z_{T 2}-Z_{T 3}\right)\right]
\end{gathered}
$$

If $i=3,4$

$$
\begin{gathered}
j_{i, 4}=\left(Y_{T 2}-p_{Y}\right)\left(\frac{\partial L_{i}}{\partial X_{T 2}} \sin \gamma+\frac{\partial L_{i}}{\partial Z_{T 2}} \cos \gamma\right)-\frac{\partial L_{i}}{\partial Y_{T 2}} \frac{a}{\sqrt{3}} \sin \beta \sin \delta \\
j_{i, 5}=\left(Z_{T 2}-p_{Z}\right) \frac{\partial L_{i}}{\partial X_{T 2}}-\left(X_{T 2}-p_{X}\right) \frac{\partial L_{i}}{\partial Z_{T 2}}
\end{gathered}
$$




$$
j_{i, 6}=\frac{1}{\sqrt{3}}\left[\frac{\partial L_{i}}{\partial X_{T 2}}\left(X_{T 3}-X_{T 1}\right)+\frac{\partial L_{i}}{\partial Y_{T 2}}\left(Y_{T 3}-Y_{T 1}\right)+\frac{\partial L_{i}}{\partial Z_{T 2}}\left(Z_{T 3}-Z_{T 1}\right)\right]
$$

If $i=5,6$

$$
\begin{aligned}
& j_{i, 4}=\left(Y_{T 3}-p_{Y}\right)\left(\frac{\partial L_{i}}{\partial X_{T 3}} \sin \gamma+\frac{\partial L_{i}}{\partial Z_{T 3}} \cos \gamma\right)-\frac{\partial L_{i}}{\partial Y_{T 3}} \frac{a}{\sqrt{3}} \sin \beta \sin \left(\delta-\frac{\pi}{3}\right) \\
& j_{i, 5}=\left(Z_{T 3}-p_{Z}\right) \frac{\partial L_{i}}{\partial X_{T 3}}-\left(X_{T 3}-p_{X}\right) \frac{\partial L_{i}}{\partial Z_{T 3}} \\
& j_{i, 6}=\frac{1}{\sqrt{3}}\left[\frac{\partial L_{i}}{\partial X_{T 3}}\left(X_{T 1}-X_{T 2}\right)+\frac{\partial L_{i}}{\partial Y_{T 3}}\left(Y_{T 1}-Y_{T 2}\right)+\frac{\partial L_{i}}{\partial Z_{T 3}}\left(Z_{T 1}-Z_{T 2}\right)\right] \\
& \mathbf{J}=\left[\begin{array}{llllll}
\partial L_{1} / \partial X_{T 1} & \partial L_{1} / \partial Y_{T 1} & \partial L_{1} / \partial Z_{T 1} & j_{1,4} & j_{1,5} & j_{1,6} \\
\partial L_{2} / \partial X_{T 1} & \partial L_{2} / \partial Y_{T 1} & \partial L_{2} / \partial Z_{T 1} & j_{2,4} & j_{2,5} & j_{2,6} \\
\partial L_{3} / \partial X_{T 2} & \partial L_{3} / \partial Y_{T 2} & \partial L_{3} / \partial Z_{T 2} & j_{3,4} & j_{3,5} & j_{3,6} \\
\partial L_{4} / \partial X_{T 2} & \partial L_{4} / \partial Y_{T 2} & \partial L_{4} / \partial Z_{T 2} & j_{4,4} & j_{4,5} & j_{4,6} \\
\partial L_{5} / \partial X_{T 3} & \partial L_{5} / \partial Y_{T 3} & \partial L_{5} / \partial Z_{T 3} & j_{5,4} & j_{5,5} & j_{5,6} \\
\partial L_{6} / \partial X_{T 3} & \partial L_{6} / \partial Y_{T 3} & \partial L_{6} / \partial Z_{T 3} & j_{6,4} & j_{6,5} & j_{6,6}
\end{array}\right]
\end{aligned}
$$

\section{Reference}

[1] Merlet JP. Parallel Robots. Dordrecht: Kluwer Academic Publishers, 2000.

[2] Dasgupta B, Mruthyunjaya TS. The Stewart Platform manipulator: A review, Mechanism and Machine Theory. $2000 ; 35: 15-40$

[3] Bourbonnais F, Bigras P, Bonev IA. Minimum-time trajectory planning and control of a pick-and-place fivebar parallel robot, IEEE/ASME Transactions on Mechatronics. 2015; 20: 740-749.

[4] Pagis G, Bouton N, Briot S, Martinet P. Enlarging parallel robot workspace through Type-2 singularity crossing, Control Engineering Practice. 2015; 39: 1-11.

[5] Babaghasabha R, Khosravi MA, Taghirad HD. Adaptive robust control of fully-constrained cable driven parallel robots, Mechatronics. 2015; 25: 27-36.

[6] Zou J, Schueller JK. Adaptive backstepping control for parallel robot with uncertainties in dynamics and kinematics, Robotica. 2014. DOI: http://dx.doi.org/10.1017/S0263574714002410.

[7] Fu K, Mills JK. Robust control design for a planar parallel robot, International Journal of Robotics and Automation. 2007; 22: 139-147.

[8] Khosravi MA, Taghirad HD. Robust PID control of fully-constrained cable driven parallel robots, 
Mechatronics. 2014; 24: 87-97.

[9] Khoa LD, Truong DQ, Ahn KK. Synchronization controller for a 3-R planar parallel pneumatic artificial muscle (PAM) robot using modified ANFIS algorithm, Mechatronics. 2013; 23: 462-479.

[10] Zhao D, Li S, Gao F. Fully adaptive feedforward feedback synchronized tracking control for Stewart Platform systems, International Journal of Control, Automation, and Systems. 2008; 6: 689-701.

[11] . López-Araujo DJ, Zavala-Río A, Santibáñez V, Reyes F. Output-feedback adaptive control for the global regulation of robot manipulators with bounded inputs, International Journal of Control, Automation and Systems. 2013; 11: 105-115.

[12] Peng W, Lin Z, Su J. Computed torque control-based composite nonlinear feedback controller for robot manipulators with bounded torques, IET Control Theory \& Applications. 2009; 3: 701-711.

[13] Dixon WE, de Queiroz MS, Zhang F, Dawson DM. Tracking control of robot manipulators with bounded torque inputs, Robotica. 1999; 17: 121-129.

[14] Zergeroglu E, Dixon W, Behal A, Dawson D. Adaptive set-point control of robotic manipulators with amplitude-limited control inputs, Robotica. 2000; 18: 171-181.

[15] Li S, Wang X. Finite-time consensus and collision avoidance control algorithms for multiple AUVs, Automatica. 2013; 49: 3359-3367.

[16] Song Z, Li H, Sun K. Finite-time control for nonlinear spacecraft attitude based on terminal sliding mode technique, ISA Transactions. 2014; 53: 117-124.

[17] Lee D, Sanyal A, Butcher E, Scheeres D. Finite-time control for spacecraft body-fixed hovering over an asteroid, IEEE Transactions on Aerospace and Electronic Systems. 2015; 51: 506-520.

[18] Galicki M. Finite-time control of robotic manipulators, Automatica. 2015; 51: 49-54.

[19] Yang Y, Hua C, Ding H, Guan X. Finite-time coordination control for networked bilateral teleoperation, Robotica. 2015; 33: 451-462.

[20] Huang J, Wen C, Wang W, Song Y-D. Adaptive finite-time consensus control of a group of uncertain nonlinear mechanical systems, Automatica. 2015; 51: 292-301.

[21] Zhao D, Li S, Gao F. Finite time position synchronised control for parallel manipulators using fast terminal sliding mode, International Journal of Systems Science. 2009; 40: 829-843.

[22] Zhao D, Li S, Zhu Q. A new TSMC prototype robust nonlinear task space control of a 6 DOF parallel robotic manipulator, International Journal of Control, Automation, and Systems. 2010; 8: 1189-1197.

[23] Zhao D, Zhao Y, Li S, Zhu Q. A new terminal converging PD control for parallel robotic manipulators with bounded torque. In Proceedings of 2011 International Conference on Modelling, Identification and Control, 
Shanghai, China 2011, pp. 115-121.

[24] Kim HS, Cho YM, Lee K-I. Robust nonlinear task space control for 6 DOF parallel manipulator, Automatica. 2005; 41: 1591-1600.

[25] Khalil HK, Nonlinear Systems. $3^{\text {rd }}$ ed. New Jersey: Prentice Hall, 2002.

[26] http://www.mathworks.com/help/physmod/sm/examples/stewart-platform.html?s_tid=gn_loc_drop

[27] Bellakehal S, Andreff N, Mezouar Y, Tadjine M. Force/position control of parallel robots using exteroceptive pose measurements, Meccanica. 2011; 46(1): 195-205.

[28] Özdemir M. Removal of singularities in the inverse dynamics of parallel robots. Mechanism and Machine Theory. 2017; 107: 71-86.

[29] Lebret G, Llu K, Lewis FL. Dynamic analysis and control of a Stewart platform manipulator. Journal of Robotic Systems. 1993; 10(5): 629-655.

[30] Liu K, Lewis F, Lebret G, Taylor D. The singularities and dynamics of a Stewart platform manipulator. Journal of Intelligent and Robotic Systems. 1993; 8: 287-308 